\title{
Strong coupling of localized plasmons and molecular excitons in nanostructured silver films
}

\author{
N. I. Cade ${ }^{*}$ T. Ritman-Meer, and D. Richards \\ Department of Physics, King's College London, Strand, London WC2R 2LS, UK
}

\begin{abstract}
We report on the resonant coupling between localized surface plasmon resonances (LSPRs) in nanostructured Ag films, and an adsorbed monolayer of Rhodamine 6G dye. Hybridization of the plasmons and molecular excitons creates new coupled polaritonic modes, which have been tuned by varying the LSPR wavelength. The resulting polariton dispersion curve shows an anticrossing behavior which is very well fit by a simple coupled-oscillator Hamiltonian, giving a giant Rabisplitting energy of $\sim 400 \mathrm{meV}$. The strength of this coupling is shown to be proportional to the square root of the molecular density. The Raman spectra of R6G on these films show an enhancement of many orders of magnitude due to surface enhanced scattering mechanisms; we find a maximum signal when a polariton mode lies in the middle of the Stokes shifted emission band.

PACS numbers: 71.36.+c,71.35.-y,73.20.Mf,78.67.-n
\end{abstract}

There is currently considerable interest in the interaction between excitonic and photonic states, as a means of modifying the photophysical properties of a system. Potential novel applications include lasers, 10 optical switches ${ }^{2]}$ and sensors ${ }^{3}$ In microcavities, mixing of exciton and photon modes leads to the formation of new polaritonic states, and has been observed in both $\operatorname{organi}^{4}{ }^{4516}$ and inorganic systems. ${ }^{7}$ More recently, coupling has been observed between excitonic and plasmonic states for semiconductor heterostructures ${ }^{[8] 9]}$ Localized plasmons are the subject of many current investigations, as they can dramatically alter the optical properties of a locally situated molecule: enhancement and confinement of the excitation field has important consequences in surface enhanced Raman scattering (SERS) $\stackrel{10}{10}$ Furthermore, localized surface plasmon resonances (LSPRs) can be engineered to produce large modifications in fluorescence intensity and lifetime. ${ }^{1112}$ Due to this, the interaction between localized plasmon modes and excitonic states has been studied recently for a variety of nanostructured systems: these include nanoparticles,, 13114 nanorods, $\frac{15}{15}$ nanovoids, $\frac{16}{16}$ and subwavelength hole arrays. ${ }^{17}$ For all these systems, strong coupling is manifested as an anticrossing behavior in the dispersion curve of the plasmon mode at the energy of the uncoupled exciton mode, indicating the formation of a hybridized exciton-plasmon polariton state; the resulting mode splitting is determined by the coupling strength of the two systems.

In this work, we report on the resonant coupling between LSPRs in nanostructured silver films (NSFs), and two different excitonic states in an adsorbed dye, and we demonstrate the importance of this mechanism for SERS. The coupling strength was tuned by varying the LSPR wavelength from 450 to $750 \mathrm{~nm}$; the resulting excitonplasmon polariton peak positions are very well fit by a three-coupled-oscillator Hamiltonian, which gives a Rabisplitting energy comparable to the largest values reported to date. Raman spectra have been taken for each film at two different wavelengths, and in both cases we find a maximum signal enhancement when the middle of the
Stokes shifted emission band is resonant with a polariton mode rather than the plasmonic or molecular absorption peak.

Glass coverslips were cleaned in an acid piranha solution $\left(3: 1 \mathrm{H}_{2} \mathrm{SO}_{4} / \mathrm{H}_{2} \mathrm{O}_{2}\right)$ for 60 minutes, and then rinsed thoroughly in deionized water. Thin silver films were prepared by thermal deposition of $\mathrm{Ag}(99.99 \% \mathrm{pu}-$ rity) in a vacuum chamber at $10^{-6}$ Torr: on one coverslip the nominal thickness of silver deposited was varied from 1-12 nm, in $1 \mathrm{~nm}$ increments, as measured with a quartz crystal oscillator. The films were characterized using atomic force microscopy (Veeco Explorer): all of the films have an irregular nanostructured surface, with the average particle size increasing with nominal thickness. Extinction spectra (Perkin Elmer UV900) were taken for each NSF, and are shown unscaled in Fig. 1(a). For the films studied here, the position of the LSPR will depend on the size, shape, and density of the Ag particles, $\frac{18}{18}$ we find that the LSPR position increases approximately linearly with nominal film thickness, as shown in the inset of Fig. 1(a). Rhodamine 6G (R6G) dye was deposited onto all the films simultaneously by vacuum sublimation to produce a uniform coverage of approximately monolayer thickness. Extinction spectra were acquired again for each film and for the R6G on bare glass; these are shown in Fig. 1(b). The R6G on glass spectrum can be deconvolved into two distinct peaks at 555 and $512 \mathrm{~nm}$, which are assigned to monomer and H-dimer excitons, respectively ${ }^{[1319}$ For the molecular densities investigated here, there is no evidence of J-dimer formation. Comparing Figs. 1(a) and (b), we see a clear modification in the absorption of the combined system which cannot be accounted for with a simple combination of the constituent absorbers. ${ }^{20}$ This is due to strong coupling of the molecular and plasmonic states, resulting in the formation of hybridized exciton-plasmon polariton modes. The inset in Fig. 1.(b) shows clearly there are three polariton peaks which red-shift with increasing film thickness.

Figure 2 shows the position of the peaks in Fig. 1)(b) plotted against the bare LSPR position, for each cor- 

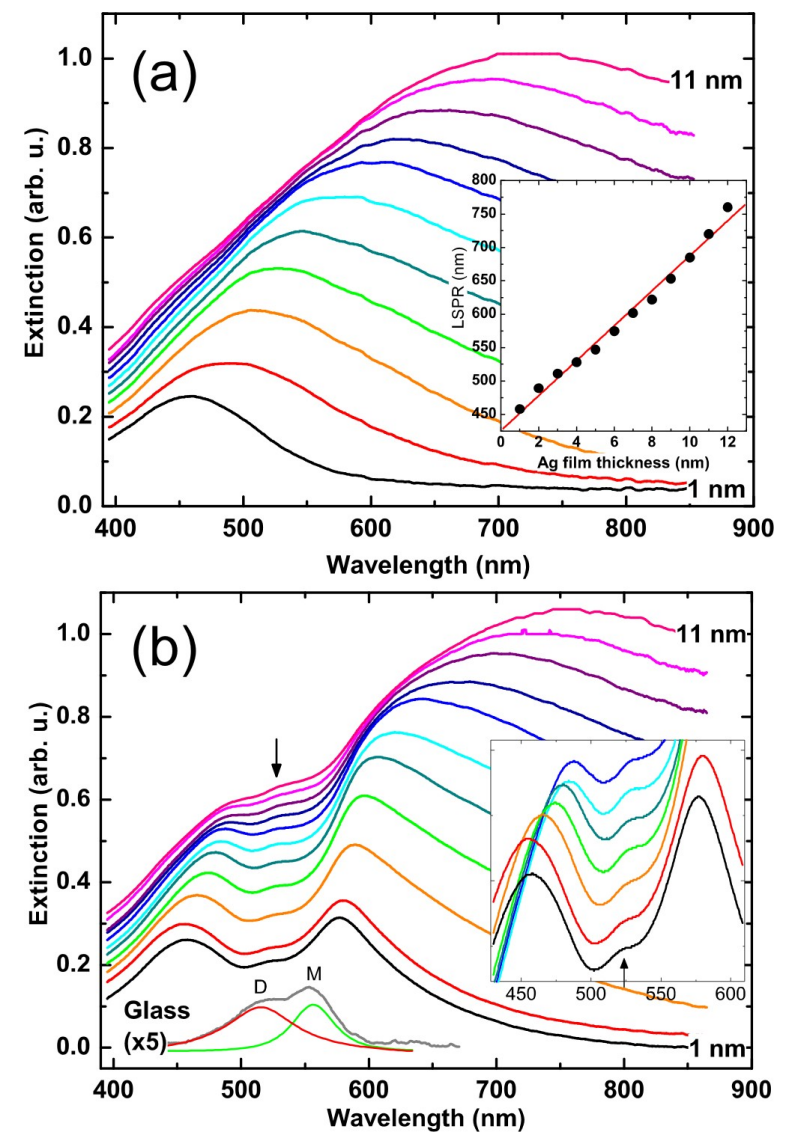

FIG. 1: (Color online)(a) Extinction spectra from bare NSFs of increasing thickness. Inset: LSPR position vs nominal film thicknesses. The solid line is a linear fit. (b) Corresponding extinction spectra after deposition of $\mathrm{R} 6 \mathrm{G}$ on the films. The short wavelength region is enlarged in the inset showing the middle polariton mode (arrow). The bottom spectrum is R6G on glass with Gaussian fits indicating monomer (M) and dimer (D) formation.

responding film thickness; the dashed and dotted lines are the plasmon and exciton positions, respectively. The data show a clear anticrossing behavior at the energies of both excitons. To better understand the coupling mechanism, the polariton dispersions were calculated using a simplified coupled oscillator model $\left[\frac{\sqrt{21}}{21}\right.$ we consider a physical system with Hamiltonian $H_{0}$ and eigenstates $|p\rangle,|m\rangle$, and $|d\rangle$ associated with the plasmon resonance and the R6G monomer and dimer excitons, respectively. The corresponding eigenvalues of the system are $E_{\mathrm{p}}, E_{\mathrm{m}}$, and $E_{\mathrm{d}}$, respectively. We assume that both excitonic eigenstates have an equal time-independent coupling $\Delta$ to the plasmonic eigenstate, and we ignore any homogeneous broadening effects; hence, the Hamiltonian can be rewritten as $H=H_{0}+\Delta$. This gives

$$
H=\left(\begin{array}{ccc}
E_{\mathrm{p}}^{\prime} & \Delta & \Delta \\
\Delta & E_{\mathrm{m}}^{\prime} & 0 \\
\Delta & 0 & E_{\mathrm{d}}^{\prime}
\end{array}\right)
$$

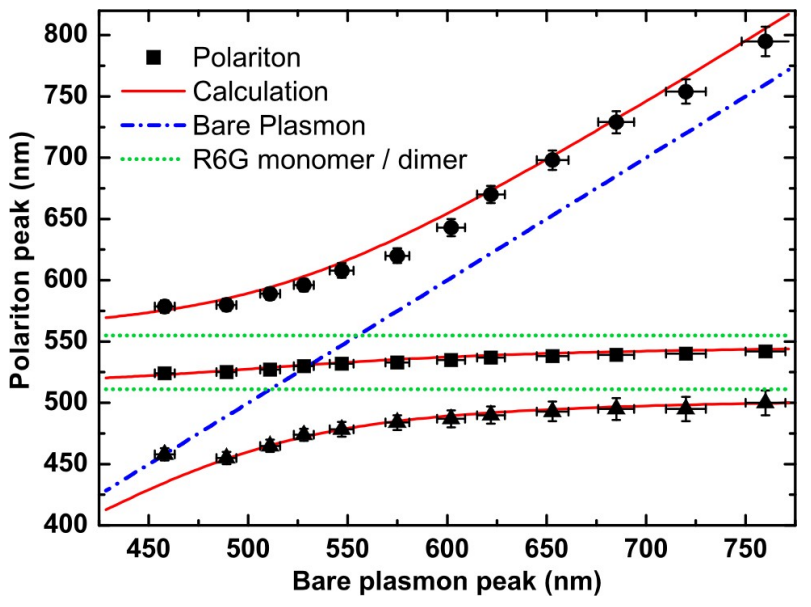

FIG. 2: (Color online) Position of the peaks in Fig. 1(b) plotted against the corresponding bare LSPR position (black symbols). Also shown are the R6G exciton peaks (dotted green), and the polariton dispersion curves calculated from Eq. 1 (solid red).

where $E_{\mathrm{p}}^{\prime}, E_{\mathrm{m}}^{\prime}$, and $E_{\mathrm{d}}^{\prime}$ are the uncoupled eigenvalues modified due to a change in the dielectric environment. For the molecular states, this change in energy only depends on the geometry of the interface rather than the material properties of the substrate itself $\frac{21}{21}$ hence, we have taken $E_{\mathrm{m}}^{\prime}$ and $E_{\mathrm{d}}^{\prime}$ directly from the peak fits of R6G on glass in Fig. 11(b). The eigenvalues of Eq. 1 were obtained numerically by assuming $E_{\mathrm{p}}^{\prime} \simeq E_{\mathrm{p}}$, and have been fitted to the data in Fig. 2 by varying only $\Delta$. We find excellent agreement with the experimental results over the whole range of wavelengths, with a coupling strength of $190 \mathrm{meV}$ corresponding to a Rabi splitting of $2 \Delta=380$ $\mathrm{meV}$. This giant splitting is comparable to those reported for organic microcavities $\sqrt[\sqrt[5]{6}]{6}$ it originates from the high oscillator strength of the dye and also the large local field amplitudes generated by surface plasmons. ${ }^{[17}$ In our case the plasmons are localized in individual Ag nanoparticles of the NSF, which will generate a much higher local field intensity than at the surface of a smooth film.22

The Rabi splitting $2 \Delta$ is expected to vary approximately as the square root of the molecular absorption. ${ }^{4|17| 23}$ To verify this, a uniform nominal $3 \mathrm{~nm}$ NSF was covered with increasing amounts of R6G; the resulting extinction spectra are shown in Fig. 3. For each level of coverage, the Rabi splitting was calculated from the polariton peak positions using the solutions of Eq. 1, and the integrated extinction was measured for the R6G on bare glass. The inset in Fig. 3 shows there is a very good agreement with the predict square root dependence. For a system of isolated nanoparticles, the spatial extent of the LSPR field is of the order of the dimensions of the nanoparticles; thus, for low coverages, increasing the density of absorbers increases the number of molecules which can couple to each LSPR. The deviation observed at the highest molecular densities is probably due to sat- 


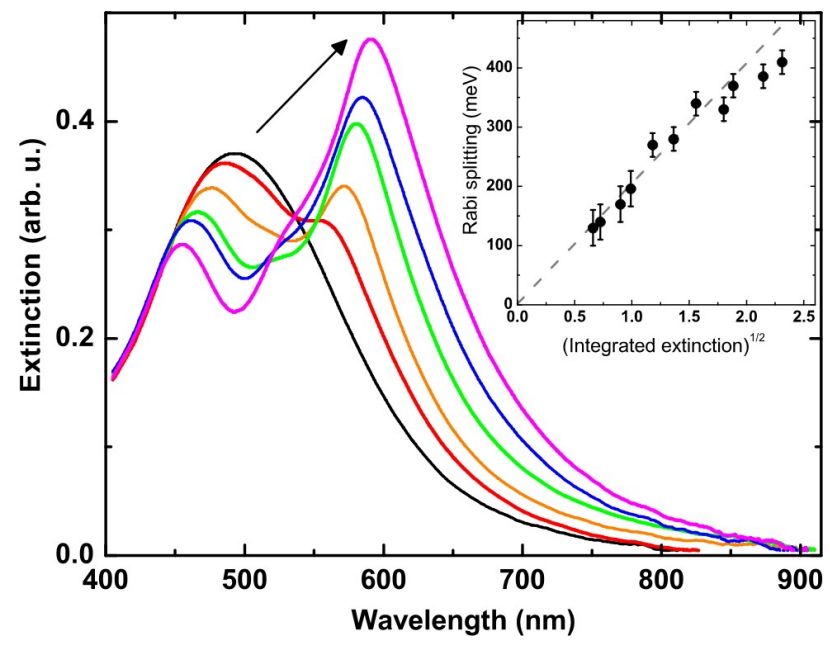

FIG. 3: (Color online) Extinction spectra from a $3 \mathrm{~nm} \mathrm{Ag} \mathrm{film}$ with increasing coverage of R6G, as indicated by the arrow. Inset: corresponding Rabi splitting $2 \Delta$, calculated from Eq. 1 , as a function of the square root of the integrated R6G extinction on glass (arbitrary units). The dashed line is a guide to the eye.

uration of electromagnetic 'hotspots' on the NSF above a surface coverage of approximately a monolayer.

The principal requirement to observe strong coupling is that the linewidths of both the excitons and plasmon must be less that the Rabi-splitting energy ${ }^{[23}$ For the NSFs investigated here, the measured LSPR linewidth will be mainly due to the inhomogeneous size and shape distribution of constituent nanoparticles. However, we assume that interactions with the covering molecules will be dominated by those nanoparticle in the peak of the distribution, with each individual nanoparticle having an intrinsic linewidth much smaller than that measured 24 For the R6G, the peak fits in Fig. 1 (b) give linewidths of $\lesssim 160 \mathrm{meV}$, which is consistent with the minimum value of the Rabi splitting for which strong coupling is observed in Fig. 3 .

To investigate further the effects of mode hybridization in the NSFs, spatially averaged Raman spectra were acquired (Renishaw 1000 microscope) for R6G on the varying thickness films described above. Excitation was at $514 \mathrm{~nm}(22 \mu \mathrm{W})$ and $647 \mathrm{~nm}(138 \mu \mathrm{W})$ with a $20 \times$ objective lens (Leica, $0.4 \mathrm{NA}$ ). Background-subtracted spectra from selected films are shown in Fig. 4 (a) for 647 $\mathrm{nm}$ excitation. No Raman signal was detectable from the R6G deposited directly onto glass. All films show a large enhancement in the R6G Raman signal due to SERS, 2526 with a strong dependence on nominal thickness and hence LSPR position. For each film, Fig. 4(b) shows the integrated intensity of the Raman peaks at $\sim 1350 \mathrm{~cm}^{-1}$ as a function of the corresponding bare LSPR position, for both excitation wavelengths. Using the total integrated intensity between $1000-1700 \mathrm{~cm}^{-1}$ gives a similar trend. For $514 \mathrm{~nm}$ excitation, a maximum enhancement occurs
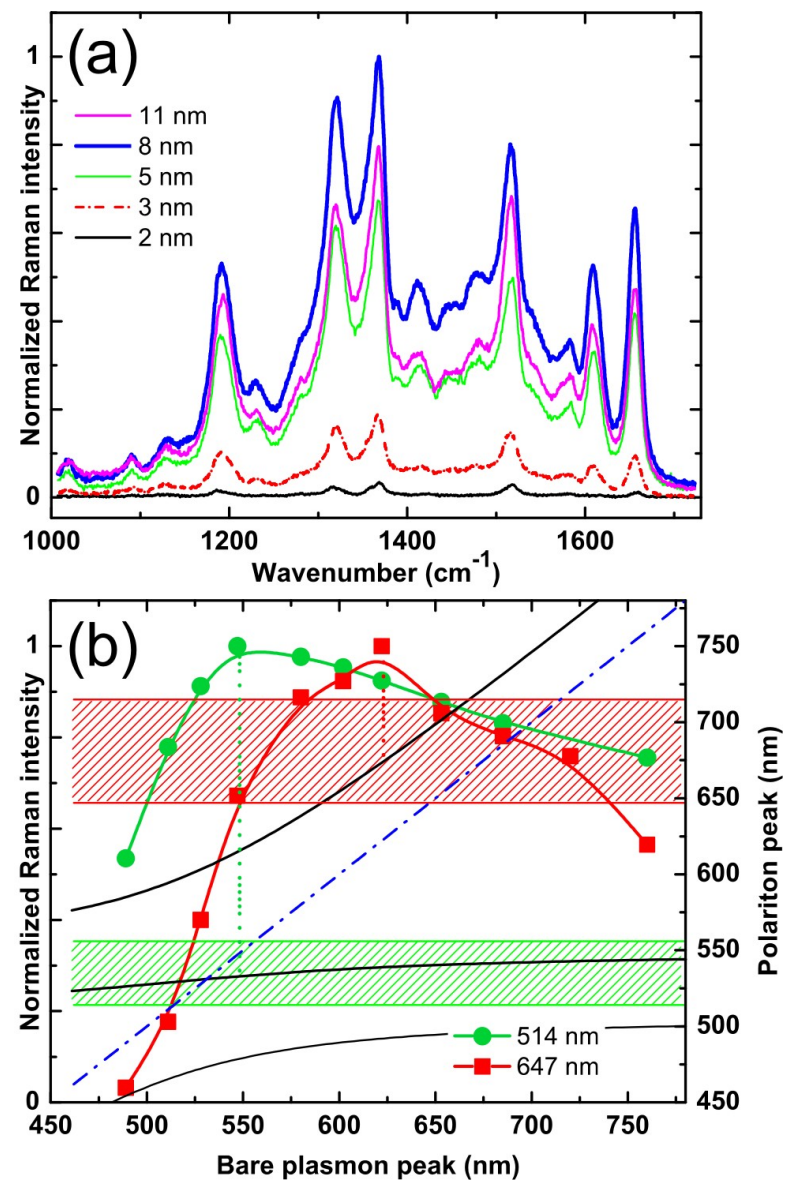

FIG. 4: (Color online)(a) Raman spectra from R6G on NSFs of increasing thickness (647 nm excitation). (b) Left axis: integrated Raman intensity from the NSFs as a function of the bare plasmon peak position. Excitation was at $514 \mathrm{~nm}$ (green circles) and $647 \mathrm{~nm}$ (red squares). Lines are guides to the eye. Right axis: polariton peak positions from Fig. 2 (solid black lines), and bare LSPR position (dashed blue line). The shaded areas correspond to the wavelength range between the incident laser and Raman peaks at $1350 \mathrm{~cm}^{-1}$. Maximum SERS enhancement occurs when a polariton mode is in the middle of this range (dotted lines).

for the $5 \mathrm{~nm}$ film with an LSPR at $550 \mathrm{~nm}$, whereas for $647 \mathrm{~nm}$ excitation, the largest signal is from the $8 \mathrm{~nm}$ film with an LSPR at $620 \mathrm{~nm}$.

In a weakly coupled system, the dominant SERS enhancement mechanism is usually amplification of the incident and scattered electric fields due to e.g. localized plasmons; the greatest enhancement has been found to occur when there is a maximum overlap between the LSPR and both laser and Raman lines.2728 The dashed line in Fig. 4(b) shows that this would occur for the $4 \mathrm{~nm}$ and $10 \mathrm{~nm}$ films with $514 \mathrm{~nm}$ and $647 \mathrm{~nm}$ excitation, respectively. When compared with the polariton peak positions in Fig. 2, Fig. 4(b) shows that in a strongly coupled system the largest SERS signal is produced when the polariton mode is halfway between the incident laser and 
the Raman emission line. There is a 30 -fold change in SERS signal for $647 \mathrm{~nm}$ excitation, as the upper polariton mode crosses the Raman enhancement region rapidly with LSPR tuning. In contrast, for $514 \mathrm{~nm}$ excitation there is only a two-fold change in SERS signal over the whole wavelength range, as the central polariton mode varies little with tuning. A similar analysis of a $450 \mathrm{~cm}^{-1}$ Raman peak close to the $647 \mathrm{~nm}$ excitation line gives a maximum signal for the $7 \mathrm{~nm}$ film (LSPR $\sim 600 \mathrm{~nm}$ ); this is consistent with the above interpretation as the upper polariton mode is closest to the laser line for this film.

In addition to the electromagnetic enhancement mechanism, SERS effects are also caused by a chemical enhancement. ${ }^{26 \mid 29}$ This mechanism has been attributed to charge transfer between the metal and molecular orbitals, and arises from a mixing of the molecular and metallic states; this can result in an additional $>10^{2}$ increase in scattering signal. In a strongly coupled system with a mesoscopic plasmonic nanostructure, such as we observe here, the two mechanisms are essen-

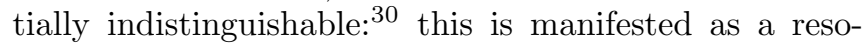
nant Raman enhancement at the wavelength of the cou- pled exciton-plasmon polariton mode rather than at the molecular or LSPR absorption maximum. The nature of the different enhancement mechanisms in SERS is still the subject of many investigations; thus, these experimental results are of particular importance in the development of a general microscopic theory for SERS.

In conclusion, nanostructured silver films have been fabricated in which the plasmon resonance can be easily tuned by varying the nominal film thickness. We have shown that strong coupling occurs between localized surface plasmons and molecular excitonic states in an adsorbed dye. The dispersion of the resulting excitonplasmon polaritonic states is in excellent agreement with a coupled-oscillator model which gives a giant Rabi splitting of $\sim 400 \mathrm{meV}$. We have verified that the coupling strength depends on the square root of the density of the absorber. SERS spectra indicate that in a strongly coupled system the greatest signal enhancement occurs when a hybridized polariton mode lies midway between the laser excitation and the Raman emission lines.

This work was supported by the EPSRC (UK).

* Electronic address: nicholas.cade@kcl.ac.uk

1 S. Christopoulos, G. B. H. von Hogersthal, A. J. D. Grundy, P. G. Lagoudakis, A. V. Kavokin, J. J. Baumberg, G. Christmann, R. Butte, E. Feltin, J.-F. Carlin, and N. Grandjean, Phys. Rev. Lett. 98, 126405 (2007).

2 D. Bajoni, E. Semenova, A. Lemaitre, S. Bouchoule, E. Wertz, P. Senellart, S. Barbay, R. Kuszelewicz, and J. Bloch, Phys. Rev. Lett. 101, 266402 (2008).

3 J. Lee, P. Hernandez, J. Lee, A. O. Govorov, and N. A. Kotov, Nat Mater 6, 291 (2007).

4 D. G. Lidzey, D. D. C. Bradley, M. S. Skolnick, T. Virgili, S. Walker, and D. M. Whittaker, Nature 395, 53 (1998).

5 P. A. Hobson, W. L. Barnes, D. G. Lidzey, G. A. Gehring, D. M. Whittaker, M. S. Skolnick, and S. Walker, Appl. Phys. Lett. 81, 3519 (2002).

6 N. Takada, T. Kamata, and D. D. C. Bradley, Appl. Phys. Lett. 82, 1812 (2003).

7 G. Khitrova, H. M. Gibbs, M. Kira, S. W. Koch, and A. Scherer, Nat Phys 2, 81 (2006).

8 J. Bellessa, C. Symonds, C. Meynaud, J. C. Plenet, E. Cambril, A. Miard, L. Ferlazzo, and A. Lemaitre, Phys. Rev. B 78, 205326 (2008).

9 P. Vasa, R. Pomraenke, S. Schwieger, Y. I. Mazur, V. Kunets, P. Srinivasan, E. Johnson, J. E. Kihm, D. S. Kim, E. Runge, G. Salamo, and C. Lienau, Phys. Rev. Lett. 101, 116801 (2008).

10 S. Nie and S. R. Emory, Science 275, 1102 (1997).

11 T. Ritman-Meer, N. I. Cade, and D. Richards, Appl. Phys. Lett. 91, 123122 (2007).

12 N. I. Cade, F. Culfaz, L. Eligal, T. Ritman-Meer, F.M. Huang, F. Festy, and D. Richards, Nanobiotechnol., 10.1007/s12030-009-9020-x (to be published 2009).

13 J. Zhao, L. Jensen, J. Sung, S. Zou, G. Schatz, and R. VanDuyne, J. Am. Chem. Soc. 129, 7647 (2007).

14 N. T. Fofang, T.-H. Park, O. Neumann, N. A. Mirin, P. Nordlander, and N. J. Halas, Nano Lett. 8, 3481 (2008).

15 W. Ni, Z. Yang, H. Chen, L. Li, and J. Wang, J. Am. Chem. Soc. 130, 6692 (2008).

16 Y. Sugawara, T. A. Kelf, J. J. Baumberg, M. E. Abdelsalam, and P. N. Bartlett, Phys. Rev. Lett. 97, 266808 (2006).

17 J. Dintinger, S. Klein, F. Bustos, W. L. Barnes, and T. W. Ebbesen, Phys. Rev. B 71, 035424 (2005).

18 R. Gupta, M. J. Dyer, and W. A. Weimer, J. Appl. Phys. 92, 5264 (2002).

19 J. E. Selwyn and J. I. Steinfeld, J. Phys. Chem. 76, 762 (1972).

20 A. M. Glass, P. F. Liao, J. G. Bergman, and D. H. Olson, Opt. Lett. 5, 368 (1980).

21 G. A. Wurtz, P. R. Evans, W. Hendren, R. Atkinson, W. Dickson, R. J. Pollard, A. V. Zayats, W. Harrison, and C. Bower, Nano Lett. 7, 1297 (2007).

22 V. A. Markel, V. M. Shalaev, P. Zhang, W. Huynh, L. Tay, T. L. Haslett, and M. Moskovits, Phys. Rev. B 59, 10903 (1999).

23 T. B. Norris, in Confined Electrons and Photons, edited by E. Burstein and C. Weisbuch (Plenum, New York, 1995), pp. 503-521.

24 C. Noguez, J. Phys. Chem. C 111, 3806 (2007).

25 A. Campion and P. Kambhampati, Chem. Soc. Rev. 27, 241 (1998).

26 M. Moskovits, J. Raman Spectrosc. 36, 485 (2005).

27 N. Felidj, J. Aubard, G. Levi, J. R. Krenn, A. Hohenau, G. Schider, A. Leitner, and F. R. Aussenegg, Appl. Phys. Lett. 82, 3095 (2003).

28 A. D. McFarland, M. A. Young, J. A. Dieringer, and R. P. Van Duyne, J. Phys. Chem. B 109, 11279 (2005).

29 B. N. J. Persson, K. Zhao, and Z. Zhang, Phys. Rev. Lett. 96, 207401 (2006).

30 B. Pettinger, J. Chem. Phys. 85, 7442 (1986). 